\title{
考虑多次反射和模式转换的电子迴 旋共振加热的射线追踪研究
}

\author{
张泽湘蒋洪英 \\ (中国科学院物理研究所, 北京)
}

在国外一些小装置(我们定义等离子体光学厚度小于 1 的装置为小装置)上所进行的电子 回旋共振加热实验 1,2$]$ 表明, 根据光学厚度估算出的能量吸收率比实验测量求得的能量吸收率 低一倍以上. 本工作就是基于波在等离子体中的实际路径(射线追踪)来计算其吸收过程, 模 拟了天线的辐射花样,考虑了波多次反射和模式转换的影响,试图解释实验结果.

\section{计算方法和所用公式}

三维射线追踪和能量沉积的计算方法同文献 [3-5]. 对于电子静电波(电子伯恩斯坦模) 我们用文献[6]中公式 (12)和(13)给出的色散关系和文献 [7]中公式 9(b) 给出的吸收系数. 所 用的等离子体温度分布 $T_{e}(\rho, \theta, \phi)$ 、密度分布 $D_{c}(\rho, \theta, \phi)$ 和磁场分布 $\left(B_{\rho}, B_{\theta}, B_{\phi}\right)$ 为下述 形式:

$$
\begin{aligned}
& D_{c}(\rho, \theta, \phi)=D_{0}\left(1-(\rho / a)^{d_{1}}\right)^{d_{2}}, \\
& T_{e}(\rho, \theta, \phi)=T_{0}\left(1-(\rho / a)^{T_{1}}\right)^{T_{i},} \\
& B_{\rho}=0, \\
& B_{\phi}=B_{0} /\left(1+\rho \cos \theta / R_{0}\right), \\
& B_{\theta}=B_{\phi} \rho /\left(R_{0} q(\rho)\right), \\
& q(\rho)=q_{0}+\left(q_{a}-q_{0}\right)\left(\rho^{2} / a^{2}\right),
\end{aligned}
$$

式中 $(\rho, \theta, \phi)$ 是准圆柱坐标, $a$ 是等离子体截面半径, $R_{0}$ 是环的大半径, $q(\rho)$ 是安全因子,下 标“ 0 ”表示截面中心的值, $d_{1} 、 d_{2} 、 T_{1}$ 和 $T_{2}$ 是常数.

\section{对 JFT-2 的计算结果}

由于 JFT-2 加热实验 ${ }^{[1]}$ 中采用的四对开端发射波导在配置上有对称性, 我们仅取其中位 于赤道面同侧且在同一截面上的两个辐射束，用五根射线来模拟每一个辐射束，其中一根为 辐射雉的中心线, 另外四根对称地分布在顶角 $16^{\circ}$ 的圆锥面上, 五根射线间的功率分配比为 $2: 1: 1: 1: 1$. 整个计算过程就是求这十根射线的轨迹及计算沿射线的能量沉积, 最后求出平均 结果. 所用标准参数组是: $R_{0}=0.90 \mathrm{~m}, a=0.25 \mathrm{~m}, B_{0}=1.0$ 式斯拉, 波频 $28 \mathrm{GHz}$, 密度参数 $d_{1}=2.0, d_{2}=1.5, D_{0}=0.075 \times 10^{20} / \mathrm{m}^{3}$ (参见式 (1)), 温度参数 $T_{1}=2.0, T_{2}^{\prime}=1.5, T_{0}=$ $600 \mathrm{eV}$ (参见式(2)), 安全因子参数 $q_{0}=1.6, q_{a}=5.0$.

本文 1985 年 1 月 24 日收到.

第 4 期

科学通报 
（A）寻常波单次通过等离子体: 标准参数下能量吸收率随场强而变的情形如下:
$B_{0}(T)$
0.9
$1.0 \quad 1.1$
1.2
吸收率 $(\%)$
32.5
51.4
48.2
14.0

根据文献 $[1]$,由实验结果得到的能量吸收率高于 $80 \%$, 而且中心场强为 1.1 式斯拉时的 吸收率明显地低于中心场强为 0.9 忒斯拉时的吸收率, 这些与上述计算结果不符. 尽管如此, 考虑实际辐射花样计算的吸收率已经比按光学厚度估算的吸收率高出 $20 \%$.

(B) 寻常波四次通过等离子体，但模式不变。我们取真空室壁的反射率为 0.98 . 按十根 射线平均的能量吸收率与场强的关系如下:
$B_{0}(T)$
0.9
1.0
1.1
1.2
吸收率 $(\%)$
$82.5 \quad 92.7$
73.1
16.5

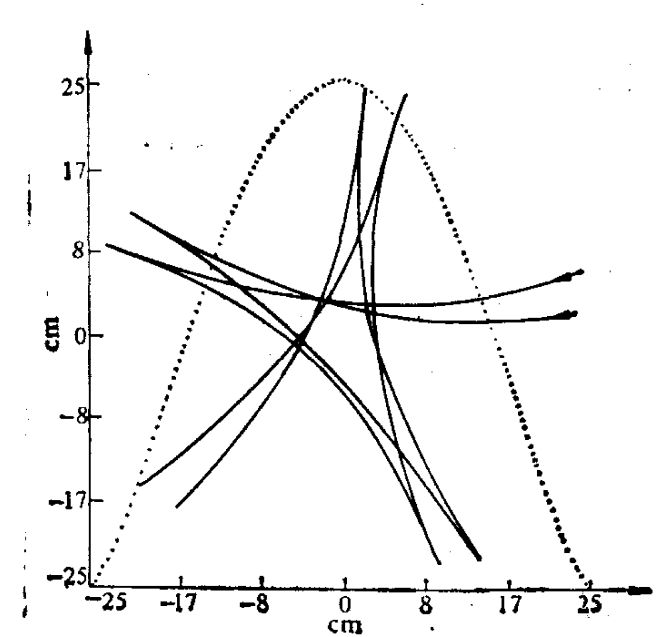

图 1a 射线轨迹在子午截面上的投影 坐标另点对应等离子体中心, 钟形 曲线示出密度分布和温度分布

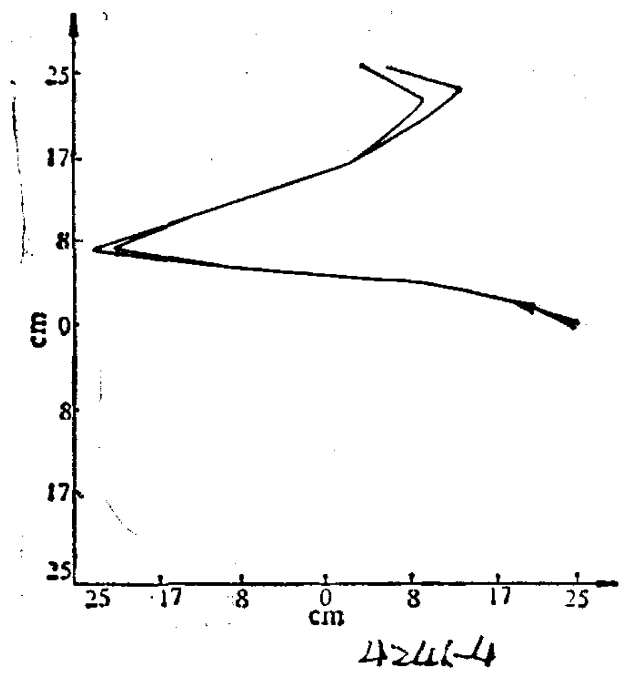

图 $1 \mathrm{~b}$ 射线轨迹在赤道面上的投影 纵轴沿环向

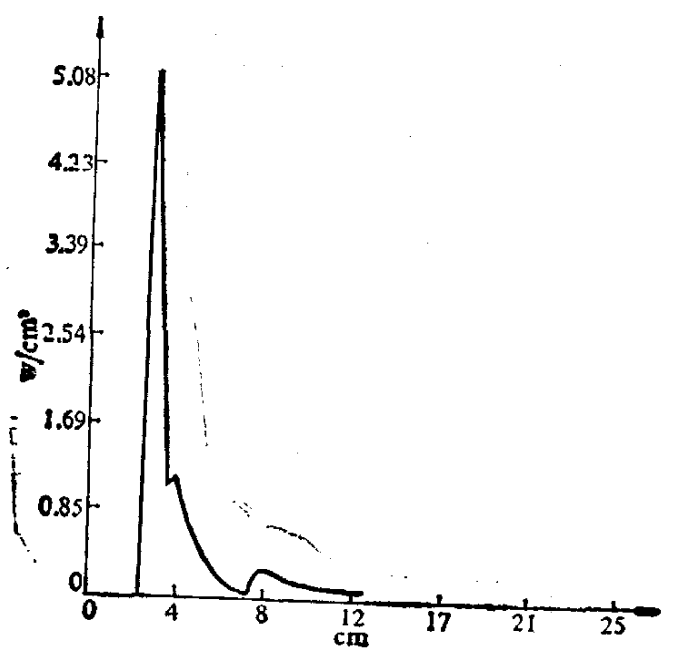

图 2 注人功率为 $100 \mathrm{kw}$ 时能量沉积分布图 $B_{0}=1.0 T$ ，横坐标原点对应等离子体中心 
这个结果与实验结果很接近. 我们将两根中心射线的轨迹示于图 $1 \mathrm{a}$ 和图 $1 \mathrm{~b}$, 将中心场强 1.0 忒斯拉下的能量沉积分布示于图 2. 考察射线轨迹后看出, 当中心场强增加或减小时共振吸 收层相对于射线轨迹的移动是不对称的. 我们也计算了仅取两个波束的中心线通过等离子体 四次的情形,其结果与十根射线的平均类似.

（C）浔常波十次通过等离子体而模式不变：这时我们仅追踪两个波束的中心线计算. 能 量吸收率随场强的变化如下:
$B_{0}(T)$
$0.9 \quad 1.1$
吸收率 $(\%)$
$98.6 \quad 98.1$

这个结果与实验结果不符,表明经过许多次反射后波的偏振改变了.

(D) 从寻常波到非常波到电子静电波(电子伯恩斯坦模)模式转换过程. 假定从环的弱场 侧注人的寻常波在通过等离子体(部份被吸收)到达内侧真空室壁被反射后变为非常波, 它到 达位于外侧边缘的高杂波共振层时假定全部转换成电子静电波(电子伯恩斯坦换)，后者又向 中心部份传布并逐渐被吸收。这个过程的计算结果如下:

$\begin{array}{lccc}B_{0}(T) & 0.9 & 1.0 & 1.1 \\ \text { 吸收率 }(\%) & 53 & 98 & 98\end{array}$

其中 $B_{0}=0.9(T)$ 时吸收仅仅是寻常波和非常波的贡献, 静电波没有有效地接近吸收层（ $\omega$ $\left.=\omega_{c}\right)$ 就偏离了. 当 $B_{0}=1.0(T)$ 和 $B_{0}=1.1(T)$ 时能量吸收率相同, 但能量沉积区域不 同. $B_{0}=1.0(T)$ 时比 $B_{0}=1.1 T$ 时能量沉积在更靠近中心的区域. 总的说来,这个假设的 波转换过程的计算结果与实验不符,这意味着所假设的模式转换过程没有发生.

(E)我们也用 TOSCA 参数 ${ }^{[1]}$ 对从弱场侧发射的韭常波二次谐波进行了计算, 考虑少数几 次反射的计算结果与实验结果接近.

\section{结论}

综合以上各种计算结果与实验的比较我们认为：（1)应当考虑整个幅射锥的平均效果和 计算少数几次反射才能给出比较准确的吸收率, 特别对小装置应如此. (2)波经过许多次反射 后其偏振会混杂化.（3)JFT-2 装置和 TOSCA 装置已有的实验结果不表明观察到了在高混杂 波共振层处从非常波到电子静电波这一模式转换过程.

\section{参考 文 献}

[1] Prator, R. and Moeller, C. P., Proc. 3rd Joint V arenna-Grenoble Int. Symp. on Heating in Toroidal Plasmas, Commission of the European Communities, Brussels, 1982, 635.

[2] Robinson, D. C. et al., ibid., 647.

[ 3 ] Zhang, Z. X., Max-Planck-Institut fur Plasmaphysik Report., IPP, Garching, 1982, 2/264.

[ 4 ] Zhang, Z. X., Proc. 5th Topical Conf. on RF Plasma Heating, Wisconsin University, Madison, 1983, C-E. 3.

[ 5 ] Wobig, H. and Zhahg, Z. X., 11th Europlan Conf. on Controlled Fusion and Plasma Physics, European Physical Society, Aachen, 1983, A22.

[6] Luca, F. D. and Maroli, C., J. Plasma Physics, 20(1978), 299.

[ 7 ] Bonatici, M. et al., Proc. 3rd Joint Varenna-Grenoble Int. Symp. on Heating in Toroidal Plasmas, Commission of the European Communities, Brussels, 1982, 691. 